\title{
Números cromossômicos em espécies de Acosmium Schott e Leptolobium Vogel (Leguminosae, Papilionoideae)
}

\author{
Rodrigo Schütz Rodrigues ${ }^{1,4}$, Andréa Macêdo Corrêa ${ }^{2}$, Eliana Forni-Martins ${ }^{3}$ e Ana Maria Goulart de Azevedo Tozzi ${ }^{3}$
}

Recebido em 9/09/2008. Aceito em 8/12/2008

RESUMO - (Números cromossômicos em espécies de Acosmium Schott e Leptolobium Vogel (Leguminosae, Papilionoideae)). O objetivo deste trabalho foi realizar a análise citotaxonômica de espécies de Acosmium Schott e Leptolobium Vogel, através da determinação de seus números cromossômicos. Foram estudadas as três espécies aceitas de Acosmium e cinco espécies de Leptolobium (representando 50\% do gênero), a partir de sementes oriundas de diferentes regiões do Brasil. As contagens mitóticas apresentadas para todas as espécies de Acosmium e para quatro espécies de Leptolobium são inéditas. Acosmium cardenasii apresentou uniformemente $2 \mathrm{n}=18$, enquanto que em A. diffusissimum foram encontradas no mesmo meristema $2 \mathrm{n}=18,24$ e 32 e em A. lentiscifolium $2 \mathrm{n}=18$ e 32. Para Leptolobium, o número cromossômico das espécies estudadas foi de $2 \mathrm{n}=18$, confirmando uma contagem mitótica anterior para L. dasycarpum. Os resultados obtidos evidenciaram homogeneidade no número cromossômico de Acosmium e Leptolobium, confirmando x $=9$ como o número cromossômico básico em ambos os gêneros. Portanto, o número cromossômico não é um caráter taxonômico utilizável na distinção entre Acosmium e Leptolobium.

Palavras-chave: aneussomatia, clado genistóide, mosaicismo, Sophoreae, taxonomia

\begin{abstract}
Chromosome numbers in species of Acosmium Schott and Leptolobium Vogel (Leguminosae, Papilionoideae)). A cytotaxonomic analysis of species of Acosmium Schott e Leptolobium Vogel was carried out, by determining their chromosome numbers. The three species of Acosmium and five species of Leptolobium (representing 50\% of the genus) were studied from seeds obtained from different regions of Brazil. Chromosome counts were new for all Acosmium species and for four Leptolobium species. For Acosmium cardenasii, $2 \mathrm{n}=18$ was constantly observed, while occurring at the same meristem were found $2 \mathrm{n}=18,24$ e 32 in A. diffusissimum and $2 \mathrm{n}=18$ e 32 in A. lentiscifolium. For Leptolobium, all studied species had $2 \mathrm{n}=18$, confirming a previous count for L. dasycarpum. The results showed that chromosome numbers of Acosmium and Leptolobium species are homogeneous, confirming the basic number $\mathrm{x}=9$ for both genera. Therefore, chromosome numbers do not provide a useful taxonomic character distinguishing Acosmium from Leptolobium.
\end{abstract}

Key words: aneussomaty, genistoid clade, mosaicism, Sophoreae, taxonomy

\section{Introdução}

Acosmium Schott pertence à tribo Sophoreae (Leguminosae Papilionoideae). Análises filogenéticas baseadas em seqüências de DNA têm sugerido que o gênero ocupa uma posição intermediária nesta subfamília, pertencendo ao clado genistóide (Pennington et al. 2001; Wojciechowski et al. 2004). O clado genistóide abrange alguns outros gêneros de Sophoreae, tais como Bolusanthus Harms, Bowdichia Kunth, Cadia Forssk., Clathrotropis Harms, Diplotropis Benth., Dicraeopetalum Harms e provavelmente Ormosia Jacks. (Pennington et al. 2001), e todos os representantes das tribos Genisteae, Euchresteae, Thermopsideae, Podalyrieae (incluindo Liparieae), Crotalarieae e Brongniartieae.

Em uma revisão de Acosmium, Yakovlev (1969) reconheceu quatro seções, com base no comprimento relativo dos lacínios e tubo do cálice, número de estames e curvatura da radícula: A. sect. Acosmium, A. sect. Leptolobium (Vogel) Yakovlev, A. sect. Mesitis (Vogel) Yakovlev e A. sect. Praeclara Yakovlev. Entretanto, recentes estudos taxonômicos e biossistemáticos (Rodrigues \& Tozzi 2007; 2008a) têm revelado que Acosmium sensu Yakovlev (1969) não compreende um grupo monofilético. Desta forma, três gêneros são reconhecidos: Acosmium, restrito às três espécies de $A$. sect. Acosmium (Rodrigues \& Tozzi 2007); Leptolobium Vogel, agrupando as dez espécies aceitas de $A$. sect.
Leptolobium e A. sect. Mesitis (Rodrigues \& Tozzi 2008b) e Guianodendron Sch. Rodr. \& A.M.G. Azevedo, abarcando a única espécie de Acosmium sect. Praeclara (Rodrigues \& Tozzi 2006).

Acosmium e Leptolobium são constituídos por arvoretas ou árvores, com flores não papilionáceas, estames livres e legumes indeiscentes, geralmente samaróides e podem ser distintos entre si com base em vários caracteres vegetativos e reprodutivos, incluindo a arquitetura dos botões florais, das inflorescências, forma dos lacínios do cálice, além da morfologia de sementes e plântulas (Rodrigues \& Tozzi 2007; 2008a). Estes gêneros apresentam espécies com distribuição exclusivamente neotropical: em Acosmium todas as espécies ocorrem no Brasil, sendo duas endêmicas e uma encontrada também na Bolívia e Paraguai; em Leptolobium, as espécies são exclusivamente sulamericanas, exceto por L. panamense (Benth.) Sch. Rodr. \& A.M.G. Azevedo, que ocorre do México até a Venezuela e Colômbia, sendo que seis espécies são encontradas somente no Brasil (Rodrigues \& Tozzi 2008b).

Apesar do grande número de espécies e de sua importância econômica, a família Leguminosae continua insatisfatoriamente conhecida citologicamente (Coleman \& DeMenezes 1980). Goldblatt (1981), em sua revisão sobre os números cromossômicos de Leguminosae, afirmou que somente cerca de $20 \%$ das espécies da família apresentam dados sobre número cromossômico, sendo que $43 \%$ dos gêneros não possuem nenhum conhecimento citológico.

\footnotetext{
1 Universidade Federal de Roraima, Centro de Ciências Biológicas e da Saúde, Boa Vista, RR, Brasil

2 Instituto de Botânica de São Paulo, Seção do Orquidário, São Paulo, SP, Brasil

Universidade Estadual de Campinas, Instituto de Biologia, Campinas, SP, Brasil

4 Autor para correspondência: rodrigo@dbio.ufrr.br
} 
Desde então, segundo Souza \& Benko-Iseppon (2004), poucos trabalhos de revisão têm sido publicados. Em Papilionoideae, recentemente destacam-se trabalhos envolvendo contagens e cariótipos de cromossomos para subsidiar a sistemática em nível genérico ou infragenérico, como os de Maciel \& Schifino-Wittmann (2002), Mendonça Filho et al. (2002), Sede et al. (2003), Flores et al. (2006) e Conterato et al. $(2006 ; 2007)$.

Em Leptolobium, existem dados sobre números cromossômicos somente para $L$. dasycarpum Vogel (sob Sweetia dasycarpa (Vogel) Benth.), com o registro de $2 n=18$ (Covas 1949) e para L. elegans Vogel (sob Acosmium subelegans (Mohlenbr.) Yakovlev), com $\mathrm{n}=9$ (Forni-Martins et al. 1992; 1995). Para Acosmium, como atualmente delimitado (Rodrigues \& Tozzi 2007), não existem registros de números cromossômicos para nenhuma de suas espécies.

O objetivo deste trabalho foi realizar a análise citotaxonômica de espécies de Acosmium e Leptolobium, mediante a determinação de seus números cromossômicos e investigar se os mesmos podem ser considerados como caráter taxonômico para individualizar os dois gêneros.

\section{Material e métodos}

Foram estudadas cinco espécies de Leptolobium (representando $50 \%$ do gênero) e as três espécies aceitas de Acosmium, cujas sementes são oriundas de diferentes regiões do Brasil (Tab. 1). Os materiais testemunho estão depositados nos herbário UEC e RB (siglas conforme Holmgren et al. 1990).

As sementes foram postas para germinar em placas de Petri à temperatura ambiente. As radículas emergidas foram coletadas e imediatamente submetidas a um pré-tratamento com solução de 8 Hidroxiquinoleína (8HQ). As radículas pertencentes às espécies de Leptolobium foram mantidas em $8 \mathrm{HQ}$ por 6 horas à $15{ }^{\circ} \mathrm{C}$ enquanto que, para as espécies de Acosmium, foram obtidos melhores resultados quando as radículas foram mantidas por 2 horas em temperatura ambiente.

Após a realização do pré-tratamento, as radículas foram fixadas em Carnoy (3 partes de etanol ou metanol absoluto : 1 parte de ácido acético glacial). A fixação foi feita à temperatura ambiente, por um período de 12 horas e estocado sob refrigeração em etanol $70 \%$. As radículas foram lavadas em água destilada e hidrolisadas com $\mathrm{HCl} 5 \mathrm{~N}$ à temperatura ambiente por 20 minutos. Posteriormente, as radículas foram esmagadas entre lâmina e lamínula, com ácido acético $45 \%$ e coradas com solução de Giemsa 2\%, por cerca de 20 minutos e montadas em resina sintética (Entelan). As lâminas foram analisadas em microscópio óptico. As melhores células foram fotografadas usando fotomicroscópio Olympus.

\section{Resultados e discussão}

As contagens cromossômicas apresentadas para Acosmium, como atualmente circunscrito, e para as espécies de Leptolobium, com exceção de L. dasycarpum, são as suas primeiras determinações mitóticas (Tab. 1).

Em Acosmium, A. cardenasii apresentou uniformemente $2 \mathrm{n}=18$ (Fig. 1A), enquanto que em A. diffusissimum foram encontradas no mesmo meristema, células com $2 \mathrm{n}=18,24 \mathrm{e}$ 32 (Fig. 1B-D) e em A. lentiscifolium $2 \mathrm{n}=18$ e 32 (Fig. 1E-F).

$\mathrm{O}$ número cromossômico encontrado nas espécies de Leptolobium foi $2 \mathrm{n}=18$ (Fig. 1G-L), confirmando a contagem realizada por Covas (1949) para L. dasycarpum. Também foi confirmado $2 \mathrm{n}=18$ para L. elegans, cujo registro anterior foi o número $\mathrm{n}=9$ (Forni-Martins et al. 1992; 1995).

Desta forma, os números cromossômicos das espécies estudadas de Leptolobium e Acosmium não contribuíram para a distinção entre estes gêneros, uma vez que ambos apresentaram $2 \mathrm{n}=18$. Entretanto, o mosaicismo cromossômico, caracterizado pela presença no mesmo meristema de células com números cromossômicos diferentes em meio às células diplóides características dos táxons, foi constatado somente em duas espécies de Acosmium.

As variações intraindividuais de número cromossômico em nível somático são denominadas polissomatia e aneussomatia, referentes à ocorrência de poliploidia e aneuploidia, respectivamente (Davide et al. 2007). Portanto, em Acosmium diffusissimum e A. lentiscifolium observou-se a ocorrência de aneussomatia. Segundo D’Amato (1997), a aneussomatia pode levar à produção ativa de aneuplóides em populações de plantas e a aneuploidia é um fator importante na especiação. Poucos casos de aneussomatia são descritos na literatura, mas esse fenômeno parece ser mais comum do que o inicialmente aceito para as plantas superiores, como, por exemplo, na família Boraginaceae (Bigazzi \& Selvi 2003). Nas duas espécies de Acosmium estudadas não se caracterizou a ocorrência de polissomatia, já que $2 n=24$ e 32 não são múltiplos perfeitos em relação a $2 \mathrm{n}=18$. Por outro lado, Berger et al. (1958) observaram polissomatia em várias leguminosas, propondo inclusive a caracterização das três subfamílias com base na freqüência de células tetraplóides

Tabela 1. Espécies de Acosmium Schott e Leptolobium Vogel (Leguminosae, Papilionoideae) analisadas, com suas respectivas coleções-testemunha procedentes do Brasil e seus números cromossômicos (2n). ES: Espírito Santo, MG: Minas Gerais, MS: Mato Grosso do Sul, PA: Pará, SP: São Paulo. *primeiras determinações mitóticas.

\begin{tabular}{ll}
\hline Táxons & Coleção-testemunha \\
\hline $\begin{array}{l}\text { A. cardenasii H.S. Irwin \& Arroyo } \\
\text { A. diffusissimum (Mohlenbr.) Yakovlev }\end{array}$ & MS, Corumbá, Damasceno 3303 (UEC) \\
$\begin{array}{l}\text { A. lentiscifolium Schott } \\
\text { L. bijugum (Spreng.) Vogel }\end{array}$ & MG, Porteirinha, Rodrigues \& Flores 1567 (UEC) \\
$\begin{array}{l}\text { L. brachystachyum (Benth.) Sch. Rodr. \& A.M.G. Azevedo } \\
\text { L. dasycarpum Vogel }\end{array}$ & ES, Linhares, Folli s.n. (UEC 146833) \\
$\begin{array}{l}\text { L. elegans Vogel } \\
\text { L. nitens Vogel }\end{array}$ & MG, Itacambira, Rodrigues \& Tozzi 1293 (UEC) \\
\hline
\end{tabular}


junto às diplóides normais. Segundo Berger et al. (1958), Papilionoideae seria a subfamília em que este fenômeno ocorreria menos freqüentemente.

A ocorrência de mosaicismo nos números cromossômicos é mais freqüentemente relatada em plantas que se reproduzem vegetativamente (Courderc et al. 1985; Jéremie et al. 2001). Por exemplo, em Artemisia dracunculus L. (Asteraceae) com 2n = 90, Kreitschitz \& Vallès (2003) relataram aneussomatia $(2 n=87,88,89$ e 90$)$ e revisaram dados sobre seu sistema reprodutivo, citando a ocorrência de anormalidades meióticas e reprodução preferencial por estolões. Em Acosmium diffusissimum foi verificada a ocorrência de uma semente poliembriônica em um lote de 20 sementes (Rodrigues \& Tozzi 2008a). A poliembrionia é encontrada em algumas espécies do cerrado e caatinga (Goldenberg \& Shepherd 1998; Salomão \& Allem 2001) e pode indicar a presença de apomixia. Desta forma, a ocorrência de poliembrionia associada à aneussomatia
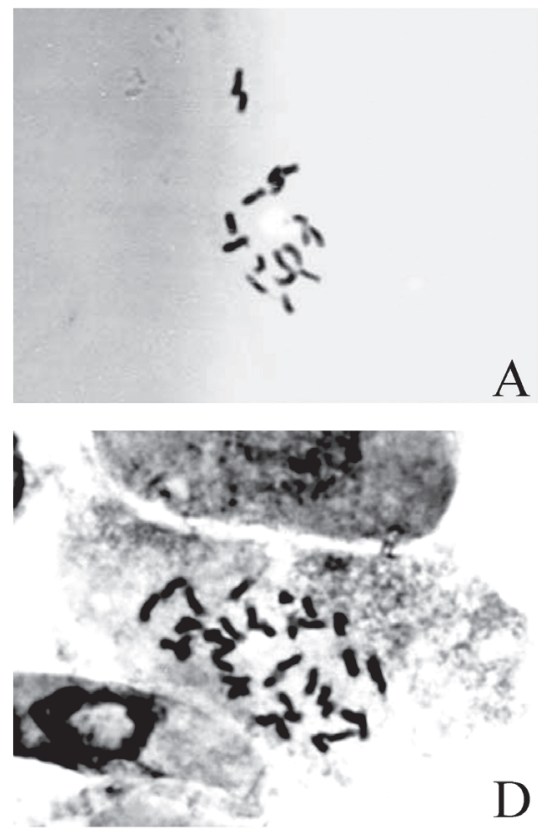

A

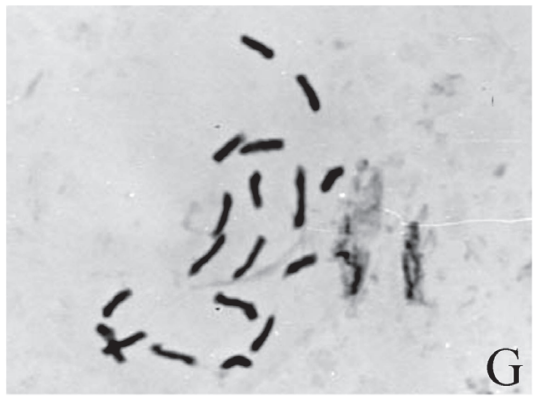

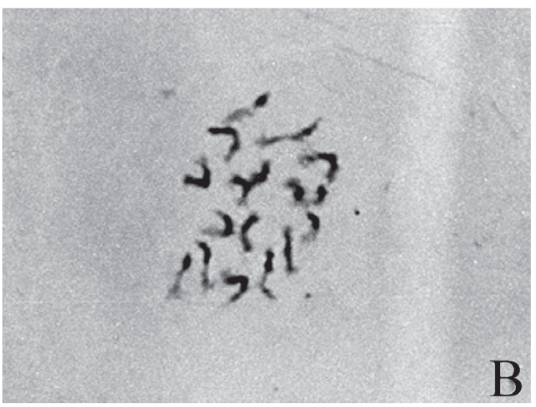

B

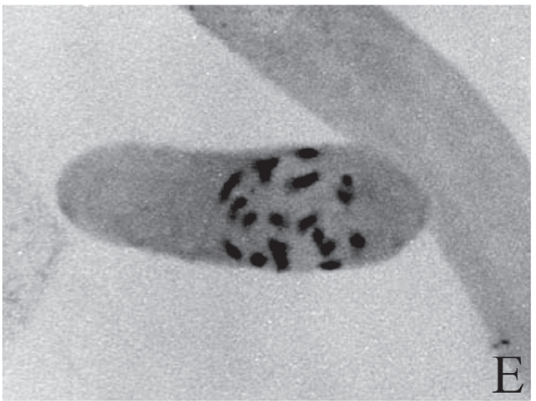

$\hat{\mathrm{E}}$

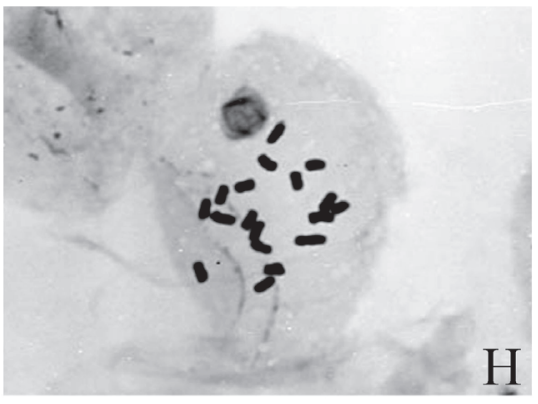

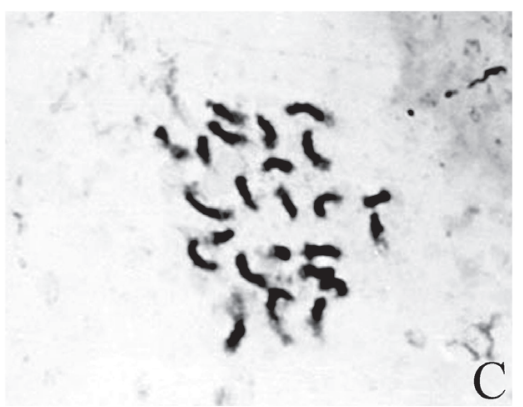

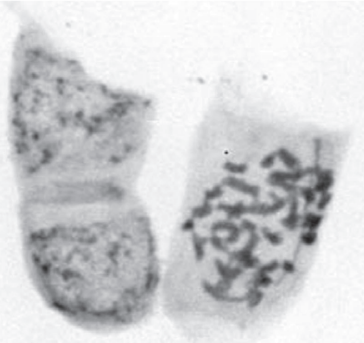

F
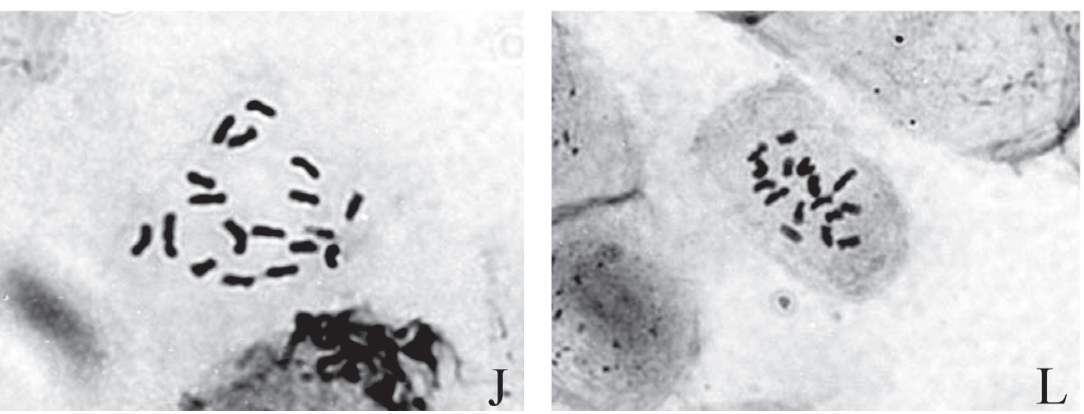

Figura 1. Números cromossômicos somáticos de espécies de Acosmium e Leptolobium. 1A. Acosmium cardenasii H.S. Irwin \& Arroyo (2n = 18). 1B-D. Acosmium diffusissimum (Mohlenbr.) Yakovlev. 1B $(2 \mathrm{n}=18)$. 1C. célula aneussomática $(2 \mathrm{n}=24)$. 1D. célula aneussomática $(2 \mathrm{n}=32) 1 \mathrm{E}-\mathrm{F}$. Acosmium lentiscifolium Schott 10. 1E. $(2 \mathrm{n}=18)$. 1F. célula aneussomática $(2 \mathrm{n}=32)$. 1G. Leptolobium bijugum (Spreng.) Vogel $(2 \mathrm{n}=18)$. 1H. Leptolobium brachystachyum $($ Benth.) Sch. Rodr. \& A.M.G. Azevedo $(2 \mathrm{n}=18)$. 1I. Leptolobium dasycarpum Vogel $(2 \mathrm{n}=18)$. 1J. Leptolobium elegans Vogel $(2 \mathrm{n}=18)$. $1 \mathrm{~L}$. Leptolobium nitens Vogel $(2 \mathrm{n}=18)$. Escala $10 \mu \mathrm{m}$ para todas as figuras. 
sugere que $A$. diffusissimum possa fazer uso da reprodução assexuada.

Anormalidades mitóticas, como não-disjunção cromossômica, foram observadas em células somáticas de ovários jovens e em anteras de Orobanche gracilis Sm. (Orobanchaceae) e apontadas como a principal causa da aneussomatia descrita na espécie (Greilhuber \& Weber 1975). Por outro lado, nas células somáticas de meristema radicular das espécies de Acosmium e Leptolobium analisadas, não foi constatada irregularidade em nenhuma fase de mitose que possa ter refletido nos números cromossômicos. Entretanto, diversas anormalidades na microsporogênese foram observadas em Leptolobium elegans (sob Acosmium subelegans) por Forni-Martins et al. (1992). Entre estas anormalidades, foram encontradas a citomixia em célulasmãe de pólen em diacinese, com números cromossômicos variados, além da disjunção cromossômica irregular em anáfase I e telófase I.

A partir dos resultados obtidos, pode-se confirmar que o número cromossômico básico em Leptolobium seja $\mathrm{x}=9$, uma vez que a metade das espécies do gênero foi analisada citologicamente, concordando com o mencionado por Goldblatt (1981) sob a designação de Acosmium. Desta forma, fenômenos de poliploidia não parecem ter contribuído em eventos de especiação no gênero, considerando a homogeneidade nos números cromossômicos das espécies estudadas. Os números cromossômicos, juntamente com dados morfológicos (Rodrigues \& Tozzi 2007) apóiam a sinonimização das seções Leptolobium (aqui amostradas: L. dasycarpum, L. elegans e L. nitens) e Mesitis (L. brachystachyum e L. bijugum), pois não foram reveladas diferenças entre os números cromossômicos entre as espécies amostradas destas duas seções sensu Yakovlev (1969). Para Acosmium, onde todas as espécies foram amostradas, o número cromossômico básico também se mostrou $x=9$.

Atualmente, alguns gêneros arbóreos de Sophoreae são considerados elementos basais do clado genistóide (Wojciechowski et al. 2004). Entre as principais sinapormorfias mencionadas por Pennington et al. (2001) para este clado, destaca-se o número cromossômico básico $\mathrm{x}=9$, encontrado em Poecilanthe Benth. s.s. (Forni-Martins obs. pes.; Meireles \& Tozzi obs. pes.), Cyclolobium Benth. e Bowdichia Kunth. (Bandel 1974; Goldblatt 1981; Souza \& Benko-Iseppon 2004). Desta forma, os resultados obtidos neste estudo estão de acordo com os encontrados nestes gêneros. Entretanto, para estudos de evolução do número cromossômico no clado genistóide, ressalta-se a necessidade de se amostrar espécies de alguns gêneros arbóreos ainda não estudados sob este aspecto, como Diplotropis e Clathrotropis, bem como espécies neotropicais de Ormosia, cujas espécies paleotropicais analisadas apresentam x = 8 (Goldblatt 1981; Goldblatt \& Johnson 1979).

\section{Agradecimentos}

Agradecemos à Dra. Andréia S. Flores, pela leitura e sugestões; ao Dr. Geraldo Damasceno Júnior, ao Dr. Sérgio M. de Faria e ao Sr. Domingos Folli, pela disponibilidade no fornecimento de sementes; à Companhia Vale do Rio Doce por permitir coletas na Reserva Florestal de Linhares; aos dois assessores anônimos, pela revisão crítica e sugestões; à FAPESP (proc. 00/09429-0) pela bolsa de Doutorado concedida ao primeiro autor junto ao Programa de Pós-Graduação em Biologia Vegetal, Instituto de Biologia.

\section{Referências bibliográficas}

Bandel, G. 1974. Chromossome numbers and evolution in the Leguminosae. Caryologia 27: 17-32.

Berger, C.A.; Witkus, E.R. \& McMahon, R.M. 1958. Cytotaxonomic studies in Leguminosae. Bulletin of Torrey Botanical Club 85: 405-410.

Bigazzi, M. \& Selvi, F. 2003. Chromosome variation in Anatolian species of Nonea Medik. (Boraginaceae), with special references to endemics and $N$. persica. Caryologia 56: 509-519.

Coleman, J.R. \& DeMenezes, E.M. 1980. Chromossome numbers in Leguminosae from the state of São Paulo, Brazil. Rhodora 82: 475-481

Conterato, I.F.; Miotto, S.T.S. \& Schifino-Wittmann, M.T. 2007. Chromosome number, karyotype and taxonomic considerations on the enigmatic Sellocharis paradoxa Taubert (Leguminosae, Papilionoideae, Genisteae). Botanical Journal of the Linnean Society 155: 223-226.

Conterato, I.F. \& Schifino-Wittmann, M.T. 2006. New chromosome numbers, meiotic behaviour and pollen fertility in American taxa of Lupinus L. (Leguminosae): contribution to taxonomy and evolutionary studies. Botanical Journal of the Linnean Society 150: 229-240.

Courderc, H.; Gorenflot, R.; Moret, J. \& Siami, A. 1985. Caractéristiques et conséquences de la variation chromosomique chez I'Ornithogalum divergens Boreau. Bulletin de la Societé botanique de France, Actualités botaniques 132: 63-71.

Covas, G. 1949. Estudios cariológicos en antófitas, III. Darwiniana 9: $158-162$.

D'Amato, F. 1997. Role of somatic mutations in the evolution of higher plants. Caryologia 50: 1-15.

Davide, L.C.; Techio, V.H.; Nunes, J.D. \& Pereira, A.V. 2007. Variação cromossômica numérica em Pennisetum. Ciência e Agrotecnologia 31: 398-405.

Flores, A.S.; Corrêa, A.M.; Forni-Martins, E.R. \& Tozzi, A.M.G.A. 2006. Chromosome numbers in Brazilian species of Crotalaria L. (Leguminosae - Papilionoideae) and their taxonomic significance. Botanical Journal of the Linnean Society 151: 271-277

Forni-Martins, E.R.; Pinto-Maglio, C.A.F. \& Cruz, N.D. 1992. Biologia da reprodução em plantas de cerrado: microsporogênese. Pp. 77-82. In: Anais do VIII Congresso da Sociedade Botânica de São Paulo. Campinas 1990. Campinas, Sociedade Botânica de São Paulo.

Forni-Martins, E.R.; Pinto-Maglio, C.A.F. \& Cruz, N.D. 1995. Chromossome numbers in Brazilian cerrado plants. Revista Brasileira de Genética 18: 281-288.

Goldblatt, P. 1981. Cytology and the phylogeny of Leguminosae. Pp. 427-463. In: R.M. Polhill \& P.H. Raven (eds.). Advances in Legume Systematics. Part 1, Kew, Royal Botanic Gardens.

Goldblatt, P. \& Johnson, D.E. (eds.) 1979-. Index to plant chromosome numbers. St. Louis, Missouri Botanical Garden. Disponível em <http://mobot.mobot.org/W3T/Search/ ipcn.html>. (Acesso em: 1/08/2008). 
Goldenberg, R. \& Shepherd, G.J. 1998. Studies on the reproductive biology of Melastomataceae in "cerrado" vegetation. Plant Systematics and Evolution 211: 13-29.

Greilhuber, J. \& Weber, A. 1975. Aneusomaty in Orobanche gracilis. Plant Systematics and Evolution 124: 67-77.

Holmgren, P.K.; Holmgren, N.H. \& Barnett, L.C. 1990. Index Herbariorum, part 1: The herbaria of the world. 8 ed. New York, The New York Botanical Garden Press.

Jéremie, J.; Lobreau-Callen, D.; Courdec, H. \& Jossang, A. 2001. Une nouvelle espéce d'Echinodorus (Alismataceae) de Guadalupe (Petites Antilles). Observations palynologiques, cytogénétiques et chimiques. Adansonia 23: 191-203.

Kreitschitz, A. \& Vallès, J. 2003. New or rare data on chromosome numbers in several taxa of the genus Artemisia (Asteraceae) in Poland. Folia Geobotanica 38: 333-343.

Maciel, H.S. \& Schifino-Wittmann, M.T. 2002. First chromosome number determinations in southeastern South American species of Lupinus L. (Leguminosae). Botanical Journal of the Linnean Society 139: 395-400.

Mendonça Filho, C.V.; Forni-Martins, E.R. \& Tozzi, A.M.G.A. 2002. New chromosome counts in Neotropical Machaerium Pers. species (Fabaceae) and their taxonomic significance. Caryologia 55: 111-114.

Pennington, R.T.; Lavin, M.; Ireland, H.; Klitgaard, B.; Preston, J. \& Hu, J.-M. 2001. Phylogenetic relationships of basal Papilionoideae legumes based upon sequences of the chloroplast trnL intron. Systematic Botany 26: 537-556.

Rodrigues, R.S. \& Tozzi, A.M.G.A. 2006. Guianodendron, a new genus of Leguminosae (Papilionoideae) from South America. Novon 16: 130-133.
Rodrigues, R.S. \& Tozzi, A.M.G.A. 2007. Morphological analysis and re-examination of the taxonomic circumscription of Acosmium (Leguminosae, Papilionoideae, Sophoreae). Taxon 56: $439-452$.

Rodrigues, R.S. \& Tozzi, A.M.G.A. 2008a. Systematic relevance of seedling morphology in Acosmium, Guianodendron, and Leptolobium (Leguminosae, Papilionoideae). Brittonia 60: 287-296.

Rodrigues, R.S. \& Tozzi, A.M.G.A. 2008b. Reinstatement of the name Leptolobium Vogel (Leguminosae, Papilionoideae, Sophoreae). Taxon 57: 980-984.

Salomão, A.N. \& Allem, A.C. 2001. Polyembryony in angiospermous trees of the Brazilian cerrado and caatinga vegetation. Acta Botanica Brasilica 15: 369-378.

Sede, S.; Dezi, R.; Greizerstein, E.; Fortunato, R. \& Poggio, L. 2003. Chromosome studies in the complex Galactia-CollaeaCamptosema (Diocleinae, Phaseoleae, Papilionoideae, Fabaceae). Caryologia 56: 295-301.

Souza, M.G.C. \& Benko-Iseppon, A.M. 2004. Cytogenetics and chromosome banding patterns in Caesalpinioideae and Papilionoideae species of Pará, Amazonas, Brazil. Botanical Journal of the Linnean Society 144: 181-191.

Wojciechowski, M.F.; Lavin, M. \& Sanderson, M.J. 2004. A phylogeny of legumes (Leguminosae) based on analysis of the plastid $m a t K$ gene resolves many well-supported subclades within the family. American Journal of Botany 91: 1846-1862.

Yakovlev, G.P. 1969. A review of Sweetia and Acosmium. Notes from the Royal Botanic Garden Edinburgh 29: 347-355. 